\title{
A Heuristic Model of Vibrotactile Haptic Feedbacks Elicitation Based on Empirical Review
}

\author{
Anak Agung Gede Dharma ${ }^{1}$ and Kiyoshi Tomimatsu ${ }^{2}$ \\ ${ }^{1}$ Kyushu University, Graduate School of Design, Fukuoka, Japan \\ ${ }^{2}$ Kyushu University, Faculty of Design, Fukuoka, Japan \\ dharma.satya.utama@gmail.com, \\ tomimatu@design.kyushu-u.ac.jp
}

\begin{abstract}
We propose a novel heuristic model of vibrotactile feedbacks elicitation. The model is based on two known tactile elicitation principles, i.e. perceived tactile sensation and apparent haptic motion. Our previous studies, along with empirical reviews were used to provide an insight of how these two principles work individually. Our preceding works on the mapping of texture phase diagram of artificial vibrotactile stimuli reveals 3 main perceived vibrotactile sensation, i.e. dampness, friction, and hardness. Furthermore, we have conducted a preliminary research to observe apparent haptic motion in our proposed haptic vest interface. Our findings and the empirical reviews imply that these two haptic principles can be used concurrently to create a novel user experience.
\end{abstract}

Keywords: vibrotactile haptic feedback, heuristic model, tactile perception.

\section{Introduction}

In recent years, haptic feedback has been intensively researched as one of the important elements of multimodal human computer interaction. Haptic feedback plays indispensable roles in some user scenarios due to its unique characteristics. They include the capability of delivering information in a non-intrusive way while simultaneously exciting the cutaneous sense with rich tactile sensations. Hence, it can be effectively used to shift user's attention from the periphery to the center of attention and vice versa. Furthermore, haptic feedback can be applied for numerous purposes such as wayfinding [1], tactile mapping in augmented reality [2], or therapy [3].

Vibrotactile stimulus is one of the types of haptic stimuli, which utilizes various kinds of waveform to convey haptic stimuli to cutaneous sense [4]. The applications of vibrotactile stimuli varies from the vibration alarm in cellular phone, game controller, touchscreen feedback, etc.

In this paper, we propose a heuristic model of vibrotactile feedbacks elicitation. Two known principles in haptic feedback, i.e. perceived haptic sensation and apparent haptic motion are discussed in section 2. In addition to empirical reviews in section 2 , we have conducted researches on perceived haptic sensation (section 3) and apparent haptic motion (section 4). Furthermore, our proposed model is discussed thoroughly in section 5. Section 6 discusses about conclusion and future works. 


\section{Related Works}

\subsection{Perceived Haptic Sensations}

The mechanoreceptors beneath the skin cause a psychophysiological haptic effect, i.e. the perception of different kinds of vibrotactile sensations such as roughness, hardness, and friction [5].

These effects are caused by different amplitude thresholds of three main mechanoreceptors (i.e. SA I, FA I, and FA II). We have proposed several models to explain three major sensations that are elicited by vibrotactile stimuli $[5,6]$. In our proposed model [5], we have developed vibrotactile waveforms by the superposition of three different kinds of frequency range. Low frequency stimuli were set at $0.4-7 \mathrm{~Hz}$, medium frequency stimuli at $25-40 \mathrm{~Hz}$, and high frequency stimuli at $200-250 \mathrm{~Hz}$.

Our findings are similar to other studies about psychological mapping of perceived haptic sensations on authentic materials [7, 8]. Roughness, hardness, and friction can be identified as three main factors to describe perceived vibrotactile haptic sensations.

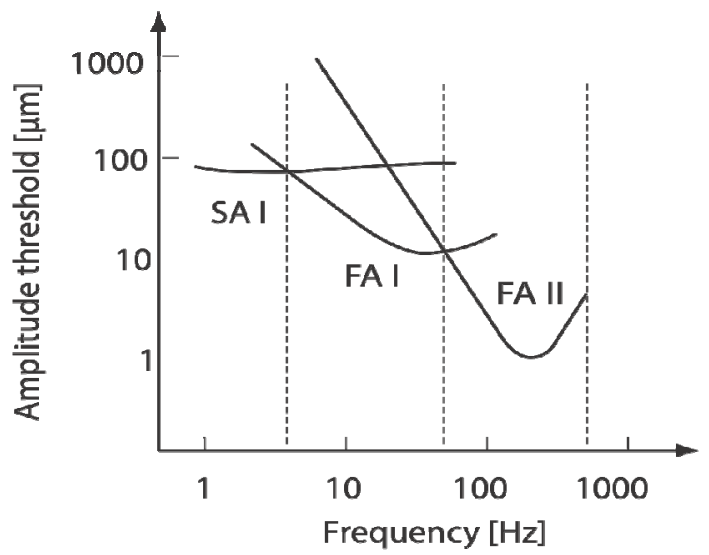

Fig. 1. Detection thresholds of vibratory stimuli (based on Bolanowski et al. [9])

\subsection{Apparent Haptic Motion}

Another aspect that needs to be taken into account when designing vibrotactile stimuli is apparent haptic motion. Apparent haptic motion is a haptic motion illusion that occurs when a series of haptic stimuli move within specific Stimuli Onset Asynchrony (SOA) [10].

Preceding works by Vaucelle et al. [2], Ertan et al. [11], and Tan et al. [12] suggest that an $\mathrm{n} \mathrm{x} \mathrm{n}$ array of actuators can be used to convey haptic information to the users, by utilizing this apparent motion. In our previous study, we have proposed a haptic vest that utilizes $5 \times 12$ arrays of vibrotactile actuators [13]. This haptic vest is designed to be worn on the torso. When a user wears the vest, it can elicit various apparent haptic motion as described by Israr et. al. Furthermore, we have observed that users react differently according to the type of haptic pattern. Their behavior can 
at least be measured by 3 variables, i.e. reaction time, apprehensibility rating, and comfort rating.
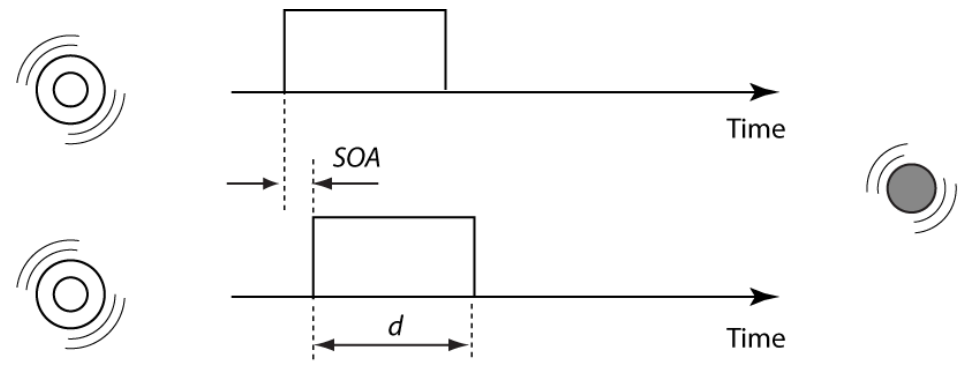

(a)
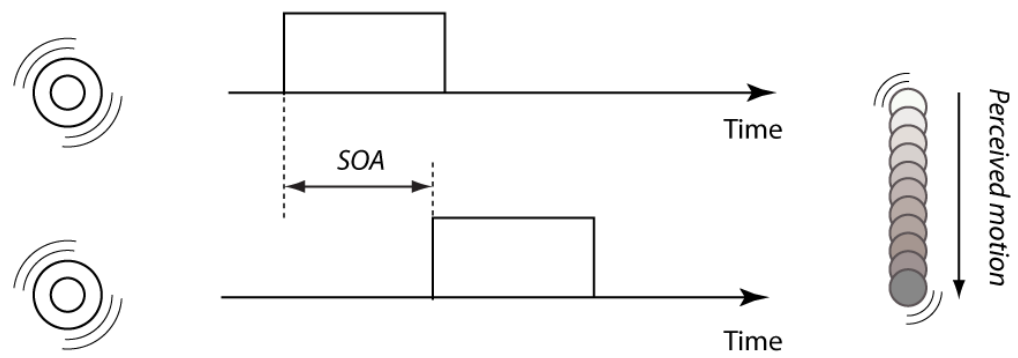

(b)
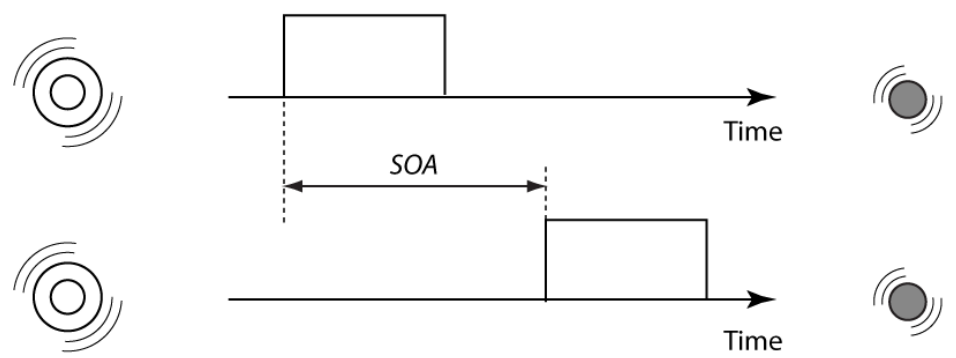

(c)

Fig. 2. Apparent tactile motion as described by Israr et al. [11]; (a) simultaneous stimulation, (b) apparent tactile motion, (c) successive stimulation

\section{Preceding Works on Perceived Haptic Sensation}

\subsection{Research Method}

Stimuli design and playback. Our vibrotactile stimuli design concept and the correlation between its variables are illustrated in Figure 3-a. Each stimulus was designed 


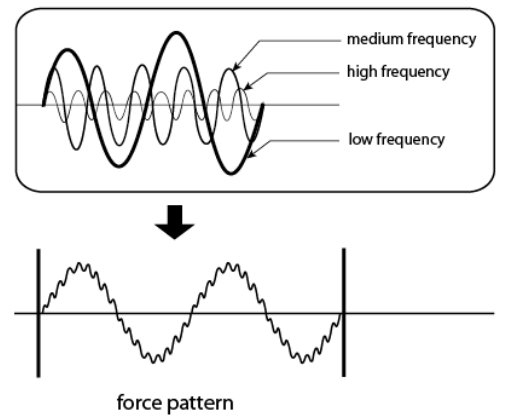

(a)

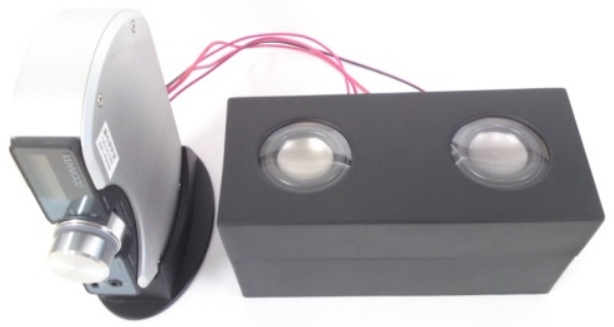

(b)

Fig. 3. (a) Three different types of frequencies to selectively stimulate SA I (low frequency), FA I (medium frequency), and FA II (high frequency); (b) A prototype to display vibrotactile stimuli that consist of a pair of vibrotactile actuators and digital amplifier

by the superposition of three haptic vibrations of different frequency ranges, i.e. the constructive interference of three different frequency ranges.

100 stimuli were generated in this experiment and evaluated by our subject participants by Semantic Differential (SD) test. The values for six variables of haptic stimulus, as described in Table 1 were chosen randomly. There were no stimuli with identical combination of those six variables. In this experiment, vibrotactile stimuli were displayed using vibrotactile actuators (Figure 3-b).

Table 1. Amplitude and frequency variables for a given force pattern

\begin{tabular}{lll||lll}
\hline $\begin{array}{l}\text { Amplitude } \\
\text { Variables }\end{array}$ & $\begin{array}{l}\text { Receptor } \\
\text { Target }\end{array}$ & $\begin{array}{l}\text { Amplitude } \\
\text { Range } \\
\text { (micron) }\end{array}$ & \begin{tabular}{ll|ll} 
Frequency \\
Variables
\end{tabular} & $\begin{array}{l}\text { Receptor } \\
\text { Target }\end{array}$ & $\begin{array}{l}\text { Frequency } \\
\text { Range (Hz) }\end{array}$ \\
\hline $\begin{array}{l}\text { Ampli- } \\
\text { tude_FA1 }\end{array}$ & $\begin{array}{l}\text { FA1 } \\
\text { (Meissner) }\end{array}$ & $0-450$ \\
\hline $\begin{array}{l}\text { Ampli- } \\
\text { tude_FA2 }\end{array}$ & $\begin{array}{l}\text { FA2 } \\
\text { (Pacinian) }\end{array}$ & $0-120$ \\
\hline $\begin{array}{l}\text { Ampli- } \\
\text { tude_SA1 }\end{array}$ & $\begin{array}{l}\text { SA1 } \\
\text { (Merkel) }\end{array}$ & $0-600$ & $\begin{array}{l}\text { FA1 } \\
\text { Frequency } \\
\text { FA2 }\end{array}$ & $\begin{array}{l}\text { FA2 } \\
\text { (Pacinian) }\end{array}$ & $200-250$ \\
\hline $\begin{array}{l}\text { Frequency } \\
\text { SA1 }\end{array}$ & $\begin{array}{l}\text { SA1 } \\
\text { (Merkel) }\end{array}$ & $0.4-7$ \\
\hline
\end{tabular}

Procedure. The experiment was conducted in a room with minimum noise and controlled temperature. The stimuli were generated by vibrotactile actuators as described in Figure 3-b. The stimuli were continuously played while the subject giving scores to Semantic Differential (SD) test. In this study, we used 7-point Likert scale SD questionnaire, both end of bipolar scale consists of "strongly felt" and "not felt at all." There were 17 onomatopoeias and 100 stimuli for SD test, therefore we had 1700 set of data from each participant [5]. 


\subsection{Results and Discussion}

We have developed a new classification method of tactile sensations to explain perceived artificial vibrotactile sensation. A texture phase diagram has been developed that can be used to explain the correlation between artificial vibrotactile stimuli and tactile perception. This experiment extracted 3 principal components of vibrotactile stimuli, i.e. dampness, friction, and hardness that account for $56.52 \%$ of overall tactile perception. This study reveals similar results with Hayakawa et al. (friction, hardness, and moisture) [8] and Hollins et al. who propose softness-hardness and roughnesssmoothness as two of the most important element in tactile sensation.

Furthermore, the explained cumulative variance is relatively low (56.52\%). Hence, it suggests that artificial vibrotactile stimuli may not adequately emulate tactile sensations that are generated by genuine physical materials. However, this result proposes a new insight towards possible applications of artificial tactile stimuli in the future.

In addition to this experiment, another experiment to measure the correlation between tactile sensations generated by physical materials and their physical properties has also been discussed [6]. We have found that roughness tactile sensation strongly correlates to surface geometrical roughness $(\mu \mathrm{m})$, softness to compliance $(\mu \mathrm{m} / \mathrm{gf})$, and stickiness to coefficient of friction $(\mu)$.

\section{Preceding Works on Apparent Haptic Motion}

\subsection{Research Method}

Participants. Sixteen subjects ( 7 males and 9 females) participated in the experiment. All subjects are undergraduate students from School of Design, Kyushu University.

Experiment Setting. Our experiment subject wears the haptic vest as shown in Figure 4-b. The experiment sequence is as follows: (1) the subject is asked to wear the haptic vest and sit throughout the experiment; (2) A stimulus is chosen randomly and exposed to the subject; (3) the stimulus is being played in a continuous loop until the subject gives a response; (4) the subject was asked to choose between four directions (back, front, left, and right) based on his/her perceived direction of the haptic stimulus; (5) The subject were asked to rate the stimulus' comfort and apprehensibility on a five step Likert scale. These sequences are repeated until the last stimulus has been selected.

Haptic Patterns. Our stimuli for the user testing experiment consist of 34 unique haptic patterns, each indicate one of four main directions (back, front, left, or right). These patterns include eight front patterns, eight back patterns, nine left patterns, and nine right patterns. Each stimulus varies on the vibration strength, haptic pattern, and the total exposure time [13]. 


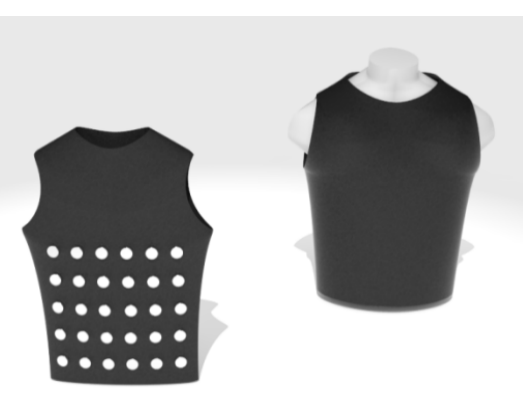

(a)

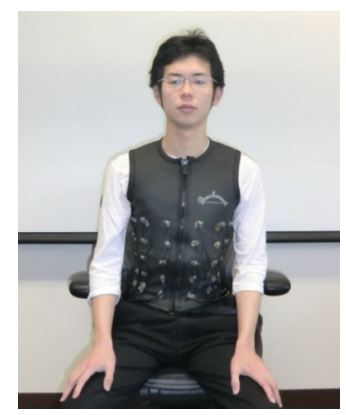

(b)

Fig. 4. (a) The design concept of our proposed wearable haptic vest, the left figure and right figure show its outer and inner view, respectively; (b) Experiment setting

\subsection{Results and Discussion}

We have developed the haptic vest as an attempt to observe to effect of apparent haptic motion. Furthermore, according to user testing, basic characteristics of perceived haptic stimulation to the users, such as comfort and apprehensibility, have been explored.

In terms of comfort and apprehensibility, we have confirmed that users evaluate back vibrations to be the most comfortable one, while front vibrations are generally evaluated as uncomfortable. Users generally preferred simple haptic patterns to complicated ones, and rate those patterns highly both in terms of comfort and apprehensibility.

In addition to apprehensibility and comfort ratings, users also reported apparent haptic motions during the experiment. Users reported apparent haptic motions for some patterns that have transitions every 0.5 seconds. However, we did not measure the effect of SOA and minimum space that is required to stimulate apparent haptic motion.

\section{Discussion: Combined Effects of Perceived Tactile Sensation and Apparent Tactile Motion}

In this paper, we propose two indispensable factors for vibrotactile haptic feedbacks design, i.e. perceived haptic sensations and apparent haptic motion. Perceived haptic sensations are cognitively evaluated by users that can be described by adjectives. They have been identified in our preceding researches [5, 6] and Hayakawa et al. [9]. These sensations are elicited by direct contact between the actuators and the skin. We have confirmed that stimulations to three different types of mechanoreceptor (SA I, FA I, and FA II) can affect perceived haptic sensations. These stimulations are caused by different ranges of mechanoreceptor frequency thresholds, as described by Bolanowski et al. [9].

Spatial and temporal settings of vibrotactile actuators affect apparent haptic motions that are perceived by users. Apparent haptic motion is elicited when vibrotactile stimuli moves spatially on the surface of the skin [10] or the actuators are positioned 
in parallel on the back and front (i.e., to generate thrusting apparent motions) [14]. Apparent haptic motion requires two or more haptic stimuli to vibrate within a specific SOA and within a specific distance.

The key characteristics, psychophysiological factors, and elicited sensations have been identified and concluded in our proposed model (Figure 5). Those characteristics and their corresponding sensations need to be taken into account for effectively designing vibrotactile haptic feedbacks in various user scenarios to create immersive user experience.

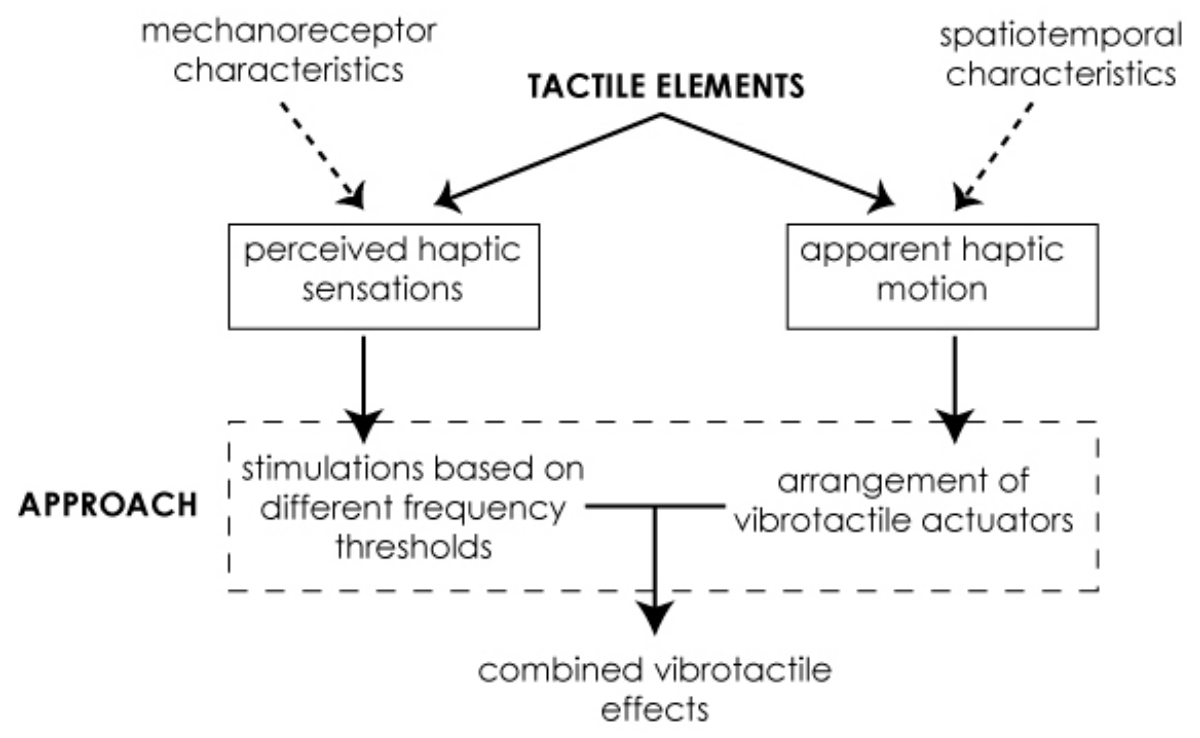

Fig. 5. Our proposed concept of the elicitation of vibrotactile effects by the combination of perceived haptic sensation and apparent haptic motion

One of prominent examples of applying these vibrotactile principles has been described in Surround Haptics by Disney Research [16]. Disney Research developed a two-dimensional haptics display mounted on the back of a chair. They fully utilize apparent haptic motion, in addition to integrating stereoscopic visuals and surround sound to simulate an immersive user experience. However, adding perceived haptic sensations into the system would create rich tactile sensation for the users, for example: the possibility to simulate rich tactile sensations by alternating the level of roughness, softness, or friction.

Moreover, we have identified other unknown factors that still need to be explored. As pointed by our proposed model in the perceived tactile sensation experiment, only $56.52 \%$ of vibrotactile stimulations could explain overall tactile sensation. Furthermore, in our haptic vest study, users reported that transitions of different haptic patterns affect their way of perceiving directions, which imply that apparent haptic motion have a role in determining user experience. 


\section{Conclusion and Future Works}

This study provides additional insights on user interactions with vibrotactile haptic feedbacks. Known physiological traits of tactile elicitations have been summarized. By combining both of perceived haptic sensations and apparent haptic motion, we suggest that rich tactile effects can be created.

As the future works, we intend to explore more vibrotactile haptic parameters to discover other unknown factors in the interaction with vibrotactile stimuli. More indepth usability tests will be performed to discover those factors and to test the validity of the proposed concept.

\section{References}

1. Heuten, X., Henze, N., Boll, S., Pielot, M.: Tactile wayfinder: a non-visual support system for wayfinding. In: Proc. 5th Nordic Conference on Human-Computer Interaction: Building Bridges, Lund, Sweden, pp. 172-181 (2008)

2. Bau, O., Poupyrev, I.: REVEL: Tactile feedback technology for augmented reality. ACM Transactions on Graphics (TOG) 31(4), 89 (2012)

3. Vaucelle, C., Bonann, L., Ishii, H.: Design of haptic interfaces for therapy. In: Proc. 27th International Conference on Human Factors in Computing Systems, Boston, USA, pp. 467-470 (2009)

4. Okamura, A.M., Dennerlein, J.T., Howe, R.D.: Vibration feedback models for virtual environments. In: Proc. IEEE International Conference on Robotics and Automation, Leuven, Belgium, pp. 674-679 (1998)

5. Dharma, A.A.G., Tomimatsu, K.: Mapping texture phase diagram of artificial haptic stimuli generated by vibrotactile actuators. In: Kurosu, M. (ed.) Human-Computer Interaction, Part IV, HCII 2013. LNCS, vol. 8007, pp. 578-586. Springer, Heidelberg (2013)

6. Dharma, A.A.G., Matsumura, Y., Tomimatsu, K.: Design of a tangible prototype for displaying hapticons. International Journal of Asia Digital Art and Design 13, 5-12 (2010)

7. Chen, X., Shao, F., Barnes, C., Childs, T., Henson, B.: Exploring relationships between touch perception and surface physical properties. International Journal of Design 3(2), 67-77 (2009)

8. Hayakawa, T., Matsui, S., Watanabe, J.: Classification method of tactile textures using onomatopoeias. Journal of the Virtual Reality Society of Japan 15(3), 487-490 (2010)

9. Bolanowski, S.J., Gescheider, G.A., Verillo, R.T., Checkosky, C.M.: Four channels mediate the mechanical aspects of touch. Journal of the Acoustical Society of America 84, 1680-1694 (1998)

10. Israr, A., Poupyrev, I.: Control space of apparent haptic motion. In: Proc. IEEE World Haptics Conference 2011, Istanbul, Turkey, pp. 457-462 (2011)

11. Ertan, S., Lee, C., Willets, A., Tan, H., Pentland, A.: A wearable haptic navigation guidance system. In: Digest of Second International Symposium on Wearable Computers, Pittsburgh, USA, pp. 164-165 (1998)

12. Tan, H.Z., Gray, R., Young, J.J., Traylor, R.: A haptic display for attentional and directional cueing. Hapticse: The Electronic Journal of Haptic Research 1(3) (2003) 
13. Dharma, A.A.G., Oami, T., Obata, Y., Yan, L., Tomimatsu, K.: Design of a wearable haptic vest as a supportive tool for navigation. In: Kurosu, M. (ed.) Human-Computer Interaction, Part IV, HCII 2013. LNCS, vol. 8007, pp. 568-577. Springer, Heidelberg (2013)

14. Ooshima, S., Fukuzawa, Y., Hashimoto, Y., Ando, H., Watanabe, J., Kajimoto, H.: Gut feelings when being cut and pierced. In: ACM SIGGRAPH 2008 New Tech Demo, p. 14 (2008)

15. Hollins, M., Faldowski, R., Rao, S., Young, F.: Perceptual dimensions of tactile surface texture: A multidimensional scaling analysis. Perception \& Psychophysics 54, 697-705 (1993)

16. Israr, A., Popyrev, I.: Exploring surround haptics displays. In: CHI 2010 Extended Abstracts on Human Factors in Computing Systems, Atlanta, USA, pp. 4171-4176 (2010) 